\title{
Bulk electronic structure of germanium studied with angle-resolved photoemission
}

\author{
J. M. Nicholls, ${ }^{*}$ G. V. Hansson, U. O. Karlsson, P. E. S. Persson, and R. I. G. Uhrberg \\ Department of Physics and Measurement Technology, Linköping Institute of Technology, 58183 Linköping, Sweden \\ R. Engelhardt \\ II Institut für Experimentalphysik der Universität Hamburg, 2000 Hamburg 50, Federal Republic of Germany \\ S. A. Flodström \\ MAX Laboratory, 22007 Lund, Sweden \\ E.-E. $\mathrm{Koch}^{\dagger}$ \\ Hamburger Synchrotronstrahlungslabor, Deutsches Electronen Synchrotron, 2000 Hamburg 52, Federal Republic of Germany
}

(Received 20 May 1985)

\begin{abstract}
The cleaved $\mathrm{Ge}(111) 2 \times 1$ surface has been studied with angle-resolved photoemission for photon energies between 10 and $35 \mathrm{eV}$. Spectra were recorded for the polarization vector of the light perpendicular and parallel to the (110) mirror plane, allowing the separation of initial states of odd and even parity, respectively. A detailed analysis of the spectra is made for several free-electron final bands. Strong features in off-normal spectra are found to correspond to a free-electron-like primary-cone final band, and several weaker secondary-cone contributions are also observed. A comparison with calculated spectra is made for normal emission and several similarities are observed between theoretical and experimental spectra, especially for transitions not described by freeelectron bands. It is suggested that the transitions contributing to the emission in the measured spectra have pronounced matrix elements mainly when the final states are close to free-electron bands, but that other final bands, which must be associated with the real band structure, contribute as well.
\end{abstract}

\section{INTRODUCTION}

For many years the techniques in the field of surface physics have been applied to the study of the bulk and surface electronic structure of germanium. In one of the earliest photoemission experiments, polarization effects were observed on the cleaved surface of germanium ${ }^{1}$ with a "directional sorting" of the electrons. This measurement was followed by a number of angle-integrated photoemission studies of the cleaved Ge(111) $2 \times 1$ surface, ${ }^{2-10}$ as well as of the annealed $\mathrm{Ge}(111) c-2 \times 8$ surface. $^{7,11}$ More recently, the technique of angle-resolved photoemission has been applied to the Ge(111) $2 \times 1$ (Refs. 12-14), $\operatorname{Ge}(111) c-2 \times 8$ (Refs. 15 and 16), and $\mathrm{Ge}(001) 2 \times 1$ (Refs. 17-20) surfaces. The Ge(111) $2 \times 1$ surface electronic structure has also been studied with partial-yield spectroscopy ${ }^{21}$ and photoemission-yield spectroscopy. ${ }^{22}$ The technique of inverse photoemission has been used to study the $\mathrm{Ge}(111) 2 \times 1$ surface, and several conduction bands are observed, ${ }^{23}$ in good agreement with calculated bands.

The bulk electronic structure has also been studied theoretically, and empirical ${ }^{24}$ as well as first-principles ${ }^{25,26}$ calculations have been performed. In interpreting the angle-resolved photoemission measurements made so far, either calculated final bands ${ }^{18}$ or a free-electron final band ${ }^{20}$ have been used.

In the present study of the cleaved $\mathrm{Ge}(111) 2 \times 1$ surface, polarized synchrotron radiation in the energy range $10-35 \mathrm{eV}$ has been used, and spectra for normal and off- normal directions of emission have been recorded for several polarization geometries. The bands from a LAPW (linearized-augmented-plane-wave) calculation ${ }^{26}$ and several free-electron final bands are used to calculate initial-state dispersions that can be compared directly with experimentally obtained dispersions. Comparison is also made with calculated photoemission spectra. Detailed analysis of the measured spectra is presented for a wide range of photon energies. From applying freeelectron final bands in interpreting the spectra, strong primary-cone $\mathrm{e}^{27}$ contributions are found, but substantial secondary-cone ${ }^{27}$ emission is also present. Some structures cannot be explained with free-electron final bands and must be associated with real band-structure effects.

\section{THEORETICAL BAND STRUCTURE}

In interpreting the measured spectra, the bands from a self-consistent-field LAPW calculation were used. ${ }^{26}$ The calculation was made within the local-density-functional (LDF) scheme, and the Hedin-Lundquist approximation ${ }^{28}$ was used for the exchange and correlation potentials. In Fig. 1 are shown the calculated bands of the main symmetry lines $X-\Gamma-L$, as well as those of the $X-L$ line in the Brillouin zone (BZ). The electron-energy bands were also calculated for a mesh of $k$ points in the (110) mirror plane and for the $(1 \overline{2} 1)$ plane in the three-dimensional Brillouin zone. An interpolation scheme was used to generate possible direct transitions between the valence and 


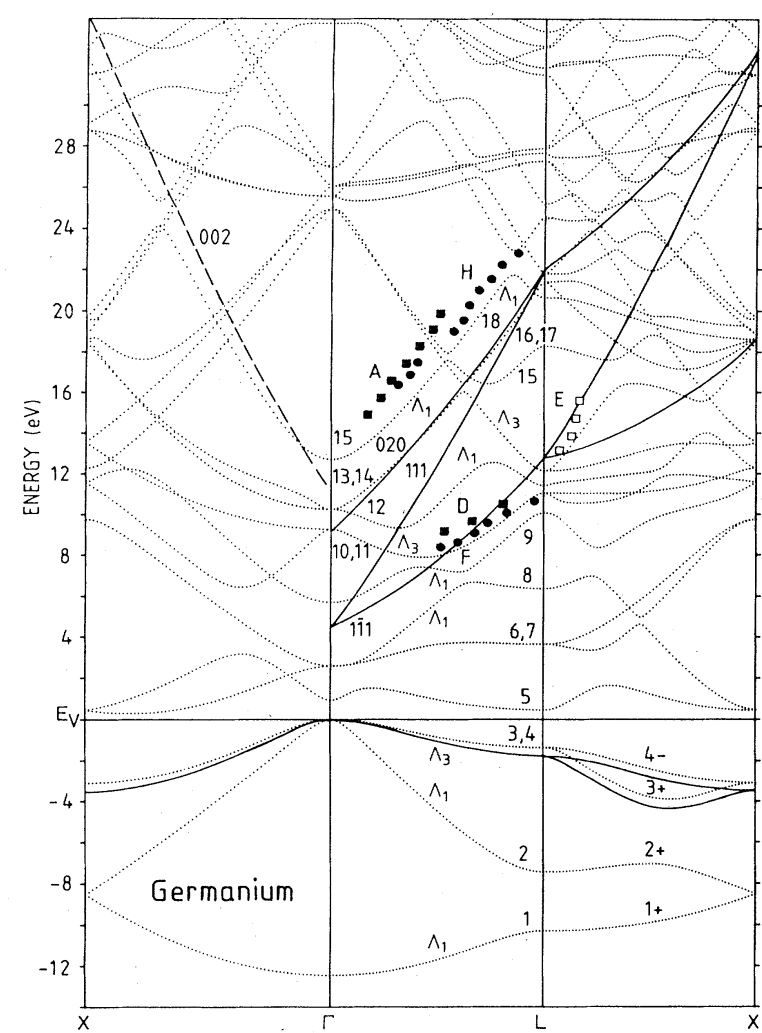

FIG. 1. Calculated band structure for germanium (Ref. 26) along the $X-\Gamma-L$ and $L-X$ lines. The free-electron final bands used in the present study and the lowered bands 3 and 4 are drawn with solid lines. The free-electron band along $\Gamma-X$ from Ref. 20 is indicated with dashed line. Experimentally found transitions from bands 2 and $3+4$, in the normal direction $\Gamma-L$, are marked with circles and squares, respectively. For details, see main text.

conduction bands, and plots were obtained for these transitions of the initial energy of the transitions as functions of $k_{\|}$relative to the surface normal. These calculated plots could then be compared directly with measured initial-state dispersions. In general, these calculated plots contain a large number of dispersion branches, even when transitions forbidden due to the symmetry of the final bands were excluded. The identification of the experimentally obtained dispersions with those obtained theoretically is therefore not unambiguous, and a more detailed knowledge of the matrix elements for these transitions is needed. As will be discussed later, lifetime effects are also likely to be of importance. In numerous cases, ${ }^{29-31}$ the application of free-electron final bands in the interpretation of photoemission spectra from both metals and semiconductors has been found useful. In a recent work this approach was successfully applied to photoemission from the $\mathrm{Si}(111) 2 \times 1$ surface, ${ }^{32}$ where transitions from the uppermost pair of valence bands to the primary free-electron final band were identified for photon energies below 21.2 $\mathrm{eV}$. In the present analysis several free-electron final bands have been used and, with the interpolation scheme mentioned above, transitions to at least three different free-electron-like final bands have been identified.
Normal-emission spectra recorded for three different polarization geometries are also compared with calculated photoemission spectra from a simplified time-reversed LEED-theory scheme. ${ }^{26}$

\section{EXPERIMENTAL DETAILS}

The angle-resolved photoemission experiment was performed in a VGADES 400 spectrometer with polarized synchrotron radiation from the DORIS II storage ring at HASYLAB, DESY. ${ }^{33}$ The electron analyzer could be rotated both horizontally and vertically, which permitted

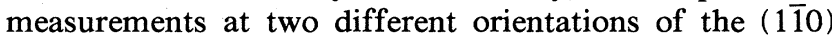
mirror plane relative to the polarization vector of the light. With the light incident within the (110) mirror plane and with the polarization vector also in that plane, spectra were recorded at angles of incidence of $\theta_{i}=45^{\circ}$ and $\theta_{i}=0^{\circ}$. (For emission angles between $0^{\circ}$ and $12.5^{\circ}$, $\theta_{i}=15^{\circ}$ was used.) These geometries will be referred to as the " $A_{\|}$" polarization geometries. As the crystal was rotated $90^{\circ}$ and the analyzer worked in the vertical mode, a smallest angle of incidence of $\theta_{i}=15^{\circ}$ could be chosen due to experimental limitations. With this geometry, the polarization vector is essentially perpendicular to the (110) mirror plane, and this will be referred to as the " $A_{\perp}$ " geometry. Off-normal-emission spectra were recorded in the (15) mirror plane with polarized light at photon energies of 10.2, 13.0, 15.0, 17.0, 19.0, and $25.0 \mathrm{eV}$. The directions [112] and [2 $\overline{1} \overline{1}]$ within the mirror plane probed in the experiment correspond to the $\bar{\Gamma}-\bar{K}$ direction in the $2 \times 1$ surface Brillouin zone (SBZ). Some emission from the dangling-bond surface state is present in the spectra, although it is strongly reduced due to the choice of near-normal incidence of the light. ${ }^{12}$ Typical values of the total-energy resolution (monochromator plus analyzer) at photon energies of 13,19 , and $25 \mathrm{eV}$ are $0.19,0.15$, and $0.27 \mathrm{eV}$, respectively, and the angular resolution was $\pm 2^{\circ}$. For all photon energies used, the position of the Fermi level was determined to an accuracy of $\pm 0.05 \mathrm{eV}$ by photoemission from the sample holder. A value of $E_{F}-E_{V}=0.1 \mathrm{eV}$ was used in calculating the initial-state dispersions. Undoped crystals with $\rho \approx 50 \Omega \mathrm{cm}$ was used. Details of the cleavage of the crystals and the determination of the domain distribution of the $2 \times 1 \mathrm{SBZ}$ have been described elsewhere. ${ }^{12,14}$ Low-energy-electron diffraction was also used to characterize the surface reconstruction. The pressure in the ultrahigh-vacuum (UHV) chamber was better than $2 \times 10^{-10}$ Torr during the experiment, and no contamination of the surface was observed.

\section{NORMAL EMISSION}

Normal-emission spectra were recorded for three different geometries, with different polarization of the light. In Figs. 2-4 normal-emission spectra are shown for the three geometries $\theta_{i}=45^{\circ}, A_{\|}, \quad \theta_{i}=15^{\circ}, A_{\|}, \quad$ and $\theta_{i}=15^{\circ}, A_{\perp}$. A large number of structures are present in the spectra and these can be divided into two categories: structures which disperse with photon energy, and features that remain at a constant binding energy for all 


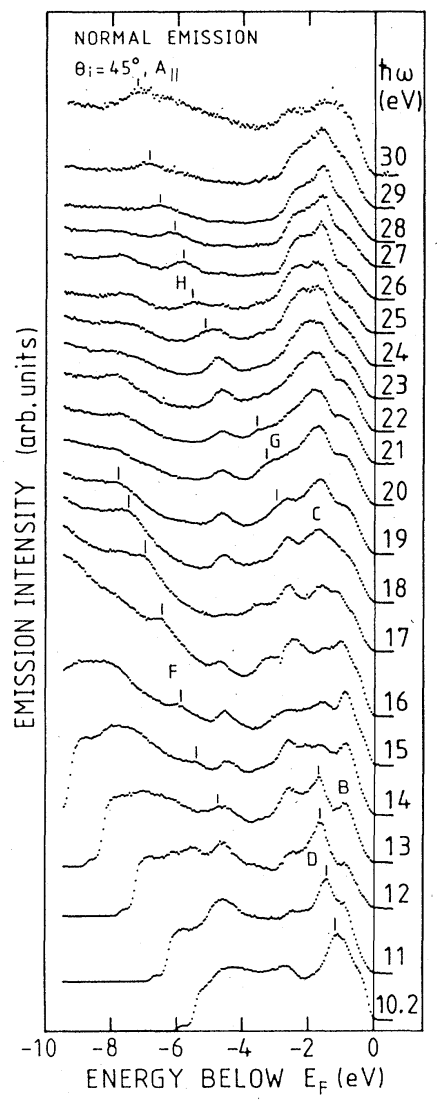

FIG. 2. Normal-emission spectra from $\mathrm{Ge}(111) 2 \times 1$ for various photon energies for the $\theta_{i}=45^{\circ}, A_{\|}$geometry. Direct transitions corresponding to the structures $D, F, G$, and $H$ are indicated in Fig. 1. For $B$ and $C$, see main text.

the photon energies where they are visible. In many cases the intensities of the emission from features in both categories change rapidly with photon energy, and it is sometimes difficult to characterize these states. Peaks which disperse with photon energy in the normal-emission spectra are generally interpreted as originating from direct transitions in the bulk, where both initial and final states are well characterized. (Dispersing structures may also be due to Auger processes in the valence bands. ${ }^{8,20}$ ) Constant initial energy features may be associated with critical points in the valence-band structure with a high density of states. In addition, such structures may also correspond to surface states, but if they are positioned within the projected bulk band structure at $\bar{\Gamma}$, a definite identification of them as surface states may be difficult. In such cases the contamination test may be used. However, the results from such measurements are often not unambiguous since the bulk features in spectra are often also influenced by foreign atoms present on the surface through, e.g., diffuse scattering of photoelectrons (see, e.g., Ref. 17).

In Fig. 1 are shown the calculated and free-electron bands used in this work. As will be discussed for the offnormal-emission measurements, it is necessary to lower bands 3 and 4 to give the best fit to the experimental data.

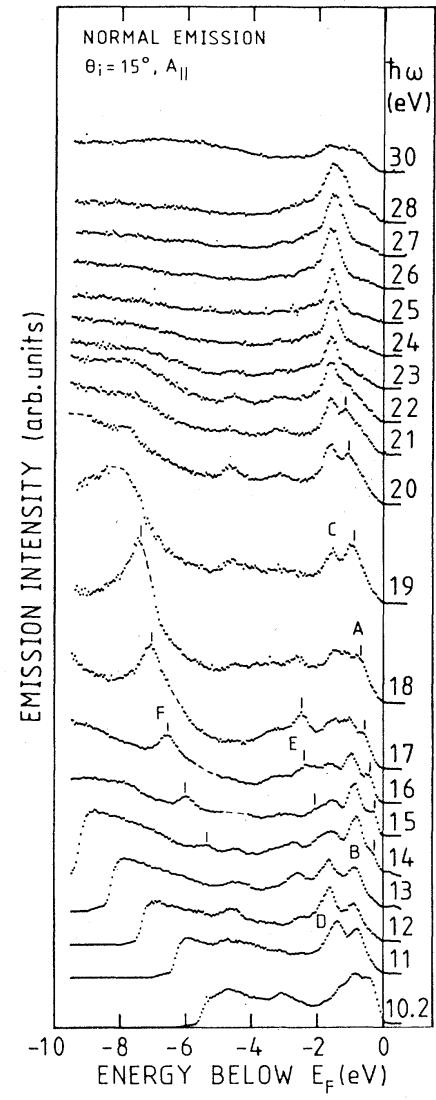

FIG. 3. Normal-emission spectra for various photon energies for the $\theta_{i}=15^{\circ}, A_{\|}$case. Structures $A$ and $F$ correspond to direct transitions and are indicated in Fig. 1 along the $\Gamma-L$ line and $E$ along the $X-L$ line. Dashed lines indicate corrections due to a disturbance from the electron analyzer.

By successively varying the positions of bands 3 and 4 and the free-electron bands, a final lowering of $0.35 \mathrm{eV}$ was found at the $L_{3}^{\prime}$ point for an inner potential of $9.5 \mathrm{eV}$.

On the cleaved $\mathrm{Ge}(111) 2 \times 1$ surface, a prominent surface state has previously ${ }^{12,14}$ been found with a $0.75-\mathrm{eV}$ dispersion throughout the $2 \times 1 \mathrm{SBZ}$. A maximum in the intensity of this surface state is observed at the $\bar{J}$ point on the $2 \times 1 \mathrm{SBZ}$ border. In normal emission this state is positioned within the bulk bands and its energy position is more uncertain $(\approx-0.75 \mathrm{eV})$. In Figs. $2-4$ some emission is observed above a binding energy of $-1 \mathrm{eV}$ in practically all spectra for the three geometries. For the case with $\theta_{i}=45^{\circ}, A_{\|}$(Fig. 2), this emission is the strongest for the three geometries, which is in accordance with the $p_{z}$ character of the dangling-bond surface state. (It has been shown that the emission from this surface state is strongly reduced for normal or near-normal incidence of the light. ${ }^{12}$ ) For binding energies of less than $1 \mathrm{eV}$, the band region close to the valence-band edge may also contribute to the emission. For the $\theta_{i}=15^{\circ}, A_{\|}$geometry (Fig. 3), a strong peak $(B)$ appears at $\approx-0.8 \mathrm{eV}$ between 10.2 and $17 \mathrm{eV}$ photon energy. This structure is probably not due to the dangling-bond surface state, but is more likely to be due to a high joint density of states along the $\Gamma-L$ line. 


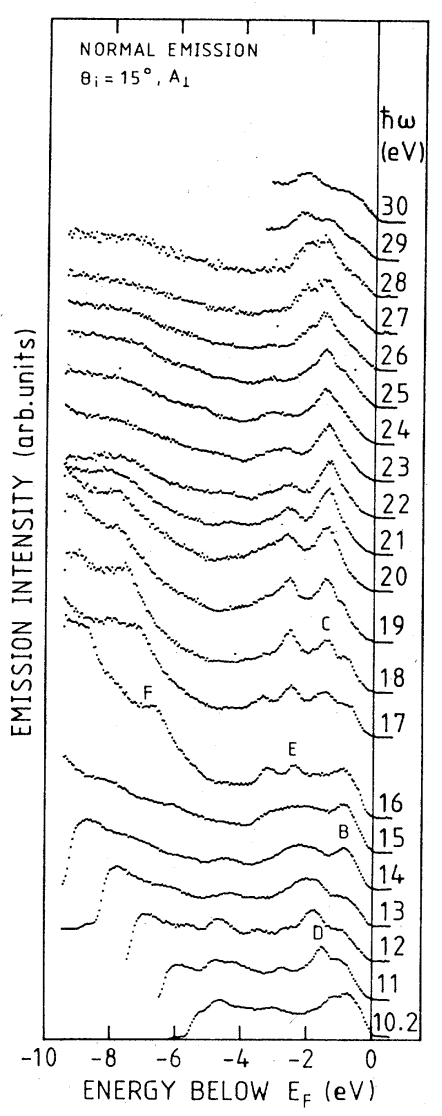

FIG. 4. Normal-emission spectra for various photon energies for the $\theta_{i}=15^{\circ}, A_{\perp}$ geometry. For a description of structures $B$, $C, D, E$, and $F$, see Figs. $1-3$ and main text.

Such a structure is also present for the other two geometries although not as strong.

In the $\theta_{i}=15^{\circ}, A_{\|}$geometry, Fig. 3, some emission is present close to the valence-band edge $(A)$ at a photon energy of $14 \mathrm{eV}$. As the photon energy increases, this structure disperses down to a minimum value of $\approx-1.1 \mathrm{eV}$ below the Fermi level for $\hbar \omega=21 \mathrm{eV}$. Assuming that peak $A$ originates from the lowered bands 3 and 4 and adding the corresponding photon energies vertically in the band structure in Fig. 1, a final band is found with a slope similar to calculated bands (bands $15+18$ ), although it is positioned $\approx 1.5 \mathrm{eV}$ above the closest calculated band with $\Lambda_{1}$ symmetry. At an initial energy of $\approx-1.65 \mathrm{eV}$, a prominent structure $(C)$ is present at most photon energies in the three series (Figs. 2-4). For photon energies of $10.2-14 \mathrm{eV}$ this structure is adjoined by a dispersing structure $(D)$. In Fig. 1 the initial energy of this structure is fitted to the lowered bands 3 and 4 . When the photon energy is added vertically in the band structure, the final

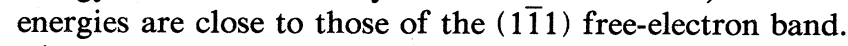
The constant-energy structure at $\approx-1.65 \mathrm{eV}(C)$ is therefore associated with emission from a high density of states close to the $L_{3}^{\prime}$ point. In fact, bands 3 and 4 are split along the $\Gamma-L$ line in the Brillouin zone by the spin-orbit interaction. The calculated value from a RAPW (relativistic-augmented-plane-wave) calculation ${ }^{26}$ of the energy split is $\approx 0.2 \mathrm{eV}$ at the $L$ point. Since bands
3 and 4 have different parity with respect to the (110) mirror plane, these bands could, in principle, be detected for the different polarization geometries of the light, $A_{\|}$ and $A_{\perp}$. For photon energies at around $23 \mathrm{eV}$, the emission from structure $C$ appears as sharp peaks for the measurements with $\theta_{i}=15^{\circ}, A_{\|}$and $A_{\perp}$ (Figs. 3 and 4). Upon closer examination, the binding energies are found to differ by approximately $0.2 \mathrm{eV}$ in this region, the lower binding energy being found for the $A_{\perp}$ case. It is notable that structure $C$ is sharp, with a high intensity of emission for photon energies around $26 \mathrm{eV}$ in all three geometries. This corresponds to transitions close to the $L$ point from bands 3 and 4 to final states in the region where a $\Lambda_{1}$ band reaches the $L$ point at $\approx 24 \mathrm{eV}$ above the valence-band edge.

In the two geometries with angle of incidence $\theta_{i}=15^{\circ}$ ( $A_{\|}, A_{\perp}$ ), a dispersing structure $(E)$ is present for initial energies between $\approx-2.0$ and $\approx-2.6 \mathrm{eV}$. In applying the previous band-mapping technique to band 2 along the $\Gamma-L$ line, a final band much too steep to be consistent with either calculated or free-electron bands is produced. While probing the $\Gamma-L$ line in normal emission for the $2 \times 1$ reconstructed surface, electrons originating from the $L-X$ line in the BZ may be scattered by a surface reciprocal-lattice vector, to escape from the surface in the normal direction. It is therefore possible that structure $E$ is associated with emission from this line. In Fig. 1 calculated bands and free-electron bands for the $L-X$ line are shown. The possible transitions corresponding to structure $E$ are marked in the band structure as transitions from the lowered band 3. The final states are then positioned close to a steeply rising calculated band as well as

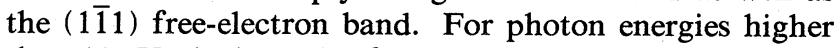
than $18 \mathrm{eV}$, the intensity from this structure decreases and remains at approximately the same initial energy for the $\theta_{i}=15^{\circ}, A_{\perp}$ geometry. For the higher photon energies some emission is observed for binding energies between -2.0 and $-2.5 \mathrm{eV}$ (see Figs. 2 and 4). Since no critical points exist in this energy range, the origin of these structures is not clear, although they have the character of constant-initial-state features. Some weak structures are also present at $\approx-3 \mathrm{eV}$ in Figs. $2-4$ and are assigned to emission from a high density of states at the $X$ point. At $\approx-4.5 \mathrm{eV}$ a constant-initial-energy state is present in many of the spectra and is associated with emission from the region around the $\Sigma_{\min }$ point along the $\Gamma-K$ line in the Brillouin zone. For all the geometries in Figs. 2-4, a structure disperses between -5 and $-8 \mathrm{eV}(F)$ for photon energies below $20 \mathrm{eV}$. This structure corresponds to direct transitions from band 2 along $\Gamma-L$ to final states close to the (111) free-electron band, in good agreement with the transitions from bands 3 and 4 to this final band (structure $D$ ). For higher photon energies, some emission is present at a binding energy of $\approx-7.8 \mathrm{eV}$, corresponding in energy to the bottom of band 2 at $L$.

Two more dispersing structures $(G$ and $H$ ) are found for the $\theta_{i}=45^{\circ}, A_{\|}$case. The corresponding direct transitions from band 2 are marked in Fig. 1 and can be seen to agree with the higher calculated bands $(15+18)$ in accordance with the transition $(A)$ from bands 3 and 4 to this band. 


\section{THEORETICAL PHOTOEMISSION SPECTRA}

In Figs. 5(a) and 5(b) theoretical normal-emission spectra for the photon-energy range 10-29 eV are shown for two different geometries of the experiment. In Fig. 5(a) spectra were calculated for the $\theta_{i}=45^{\circ}, A_{\|}$geometry and in Fig. 5(b) for the $\theta_{i}=15^{\circ}, A_{\|}$geometry. The spectra for the two geometries differ significantly only in the emission from band 2, for which a component of the electric field vector perpendicular to the surface is necessary. ${ }^{34,35}$ The calculated spectra for the geometries $\theta_{i}=15^{\circ}, A_{\perp}$ (not shown) and $\theta_{i}=15^{\circ}, A_{\|}$are almost identical, with appreciable emission from bands 3 and 4 only. In the present nonrelativistic calculation, bands 3 and 4 are degenerate along the $\Gamma-L$ line and give the same emission intensities for the two polarization geometries. The calculation was performed by assuming direct transitions and dipole matrix elements, and hence the calculated spectra do not reveal any density-of-states effects as probed by, e.g., surface photoemission. However, lifetime effects were included by Lorentzian broadening of both the initial and final states, and features with approximately constant initial energies are observed for emission to the broadened conduction bands. A description of the calculational method has been published elsewhere. ${ }^{36}$ From a closer examination of the features in the calculated spectra, the bands involved in the direct transitions can be identified. For the low-binding-energy region, structures $a, b$, and $c$ correspond to transitions from bands 3 and 4 to bands $15+18,12$, and 9 , respectively. Structures $d$, $e$, and $f$ correspond to transitions from band 2 to bands $15+18$, 12 , and 9 respectively. Several of the structures in the experimentally obtained normal-emission spectra, discussed in the preceding section, can be recognized in the calculated spectra. It is interesting to note that bands $15+18$ contribute to the emission in the calculated spectra. These bands are in close agreement with the upper final band obtained experimentally. This final band is observed for transitions from band 2 as well as from bands 3 and 4 (structures $G$ and $H$ in Fig. 2 and structure $A$ in Fig. 3). Structures $b$ and $e$ in the calculated spectra correspond to transitions to band 12 .

Similar structures are observed in the experiment, (structures $D$ and $F$ in Fig. 3), but these are found to agree better with the (111) free-electron band, which is positioned $\approx 2 \mathrm{eV}$ below band 12 . However, comparison of structures close to the valence-band edge (between experimental and theoretical spectra) is complicated, since several structures are present in the experimental spectra in this region that do not have the character of direct transitions. In practically all the spectra in Figs. 5(a) and 5 (b) some emission is present at $\approx-1.2 \mathrm{eV}$ below the Fermi level, reminiscent of the appearance of structure $C$ in Figs. $2-4$. The $L_{3}^{\prime}$ point is situated $-1.39 \mathrm{eV}$ below the valence-band maximum, and consequently the emission in the calculated spectra from bands 3 and 4 does not have the peak at an initial energy corresponding to the $L_{3}^{\prime}$ point but at a $k$ value closer to the $\Gamma$ point.

An evident result in the calculated spectra is the polarization dependence of transitions from band 2. For an angle of incidence $\theta_{i}=15^{\circ}$ almost no emission is predicted from this band. ${ }^{34,35}$ In contrast, the measured emission from this band is enhanced for the $\theta_{i}=15^{\circ}, A_{\|}$geometry (Fig. 3).

\section{OFF-NORMAL EMISSION}

In interpreting spectra recorded in off-normal directions, the previously mentioned interpolation scheme was applied to calculated initial-state bands and free-electron

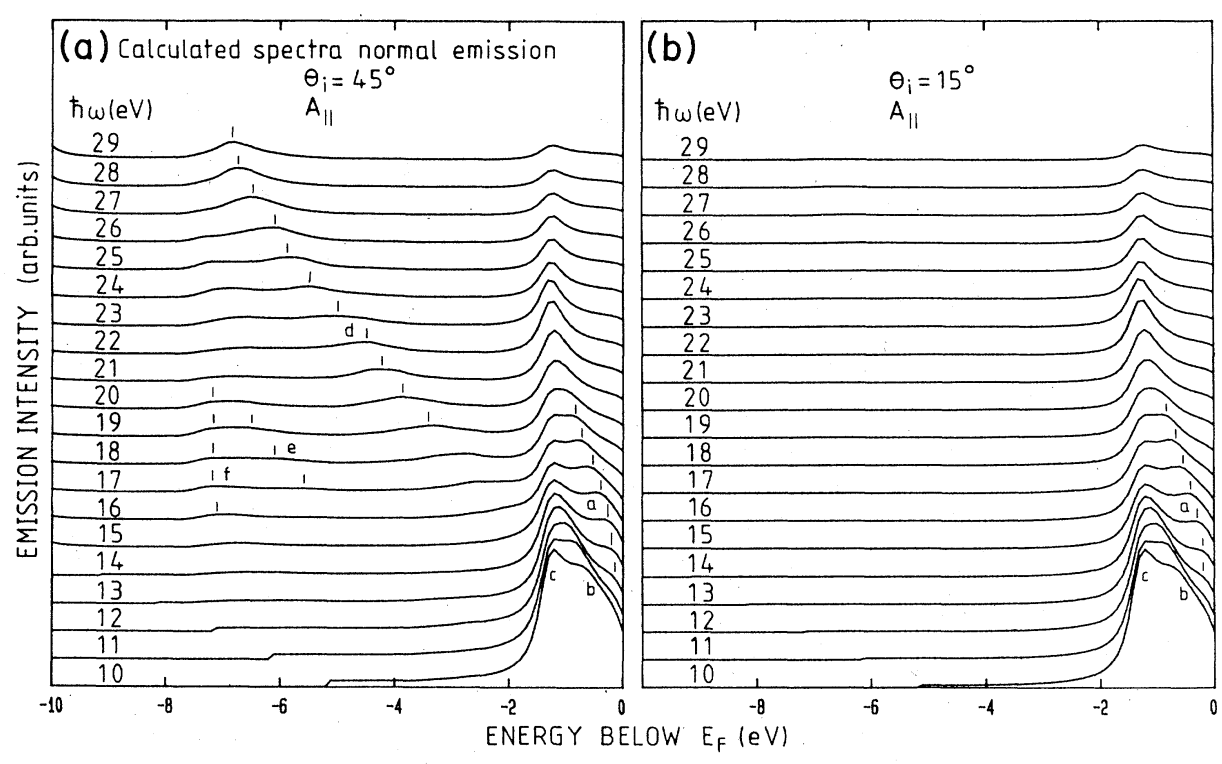

FIG. 5. Calculated photoemission spectra (Ref. 36) for Ge(111), normal emission for the (a) $\theta_{i}=45^{\circ}, A_{\|}$and (b) $\theta_{i}=15^{\circ}, A_{\|}$ geometries. Emission from bands 3 and 4 (structures $a, b$, and $c$ ) are almost identical for the two geometries, while emission from band 2 (structures $d, e$, and $f$ ) differs significantly. 
final bands. In a recent photoemission study of the $\mathrm{Si}(111) 2 \times 1$ surface, $^{32}$ a lowering by $0.45 \mathrm{eV}$ of the calculated valence bands 3 and 4 at the $L_{3}^{\prime}$ point was applied to give better agreement with observed initial energies. Similarly, a lowering of bands 3 and 4 is necessary in the present study for a best fit to experimental data. The lowering is applied in the whole plane in the BZ that is investigated and is proportional to the distance from $\Gamma$. In this case, the $L_{3}^{\prime}$ point is lowered from -1.39 to -1.74 $\mathrm{eV}$ below the valence-band edge. The lowering of bands 3 and 4 and the position of the free-electron final bands were varied successively to give the best agreement with experiment. From the alterations of the valence-band structure finally adapted, an inner potential of $9.5 \mathrm{eV}$ for the free-electron final bands was found. For an inner potential of $7.7 \mathrm{eV}$ for the free-electron bands as suggested by Hsieh et al. ${ }^{20}$ the interpolation scheme produced binding energies far too low to be consistent with the experimental data. Instead, a non-free-electron-like final band is likely to contribute to the emission in the finalband energy region studied, as shown by Nelson et al. ${ }^{18}$ for the $\Gamma-X$ line and for the $\Gamma-L$ line in the present work.

From general group-theoretical considerations, ${ }^{1}$ the al-

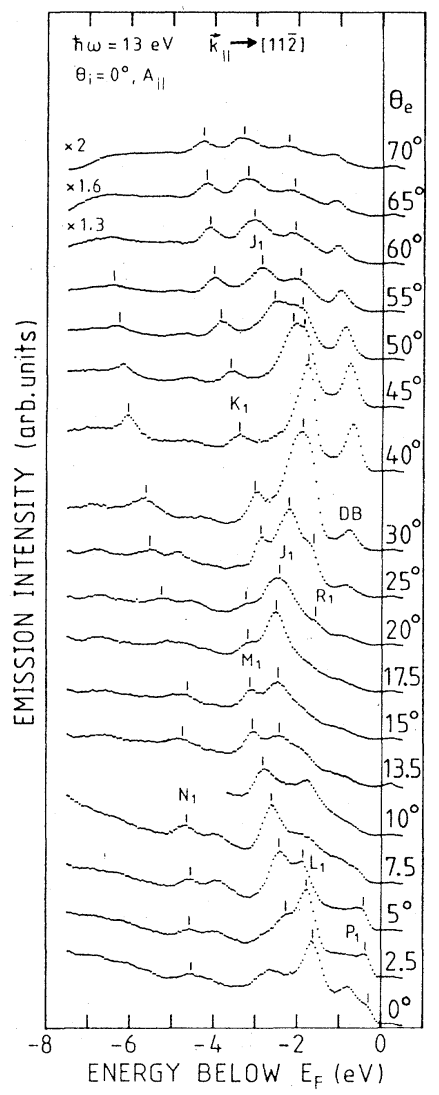

FIG. 6. Photoemission spectra for various emission angles

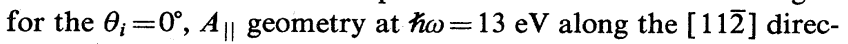
tion. For emission angles $0^{\circ}-13.5^{\circ}, \theta_{i}=15^{\circ}$ was used. Structure $J_{1}$ corresponds to emission from the even-parity band 3 to the (111) free-electron band. DB corresponds to emission from the dangling bond band. lowed transitions within a mirror plane can be determined. For the (1 $\overline{1} 0)$ mirror plane, initial states of even and odd parity are probed for the $A_{\|}$and $A_{\perp}$ geometries, respectively. In Figs. 6 and 7 spectra recorded at $\hbar \omega=13$ $\mathrm{eV}$ for the $A_{\|}$and $A_{\perp}$ geometries, respectively, are shown. The two series of spectra are markedly different due to the different orientations of the polarization vector relative to the mirror plane. In Fig. 6 the dominating feature in the spectra is a rapidly dispersing peak $\left(J_{1}\right)$ from $-2.45 \mathrm{eV}$ at $\theta_{e}=13.5^{\circ}$ to a maximum energy of -1.75 $\mathrm{eV}$ at $\theta_{e}=40^{\circ}$. For larger angles, this peak splits up into two dispersing structures. From the main structure $\left(J_{1}\right)$, a secondary structure $\left(K_{1}\right)$ also splits off at $\theta_{e}=25^{\circ}$ and disperses downwards for increasing emission angles. Some emission from the dangling-bond (DB) surface state is also present in the spectra, although the emission from this surface state is very much reduced due to the orientation of the polarization vector. ${ }^{12}$ In Fig. 7 the spectra are dominated by a slowly dispersing structure $\left(S_{1}\right)$ from $-1.0 \mathrm{eV}$ at normal emission to $-1.9 \mathrm{eV}$ at $\theta_{e}=60^{\circ}$.

In Fig. 8 the experimental initial-energy dispersions [ $\left.E_{i}\left(\mathbf{k}_{\|}\right)\right]$are compared with the theoretically predicted dispersions for several free-electron final bands. Points obtained for the $A_{\|}$and $A_{\perp}$ geometries are marked with squares and circles, respectively, while solid and open

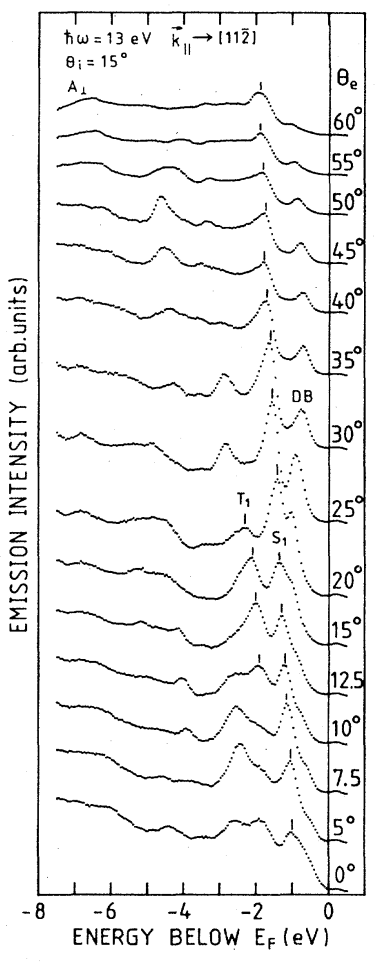

FIG. 7. Photoemission spectra for various emission angles for the $\theta_{i}=15^{\circ}, A_{\perp}$ geometry at $\hbar \omega=13 \mathrm{eV}$ along the [112] direction. Structure $S_{1}$ corresponds to emission from the oddparity band 4 to the (111) free-electron band. DB corresponds to emission from the dangling bond band. 


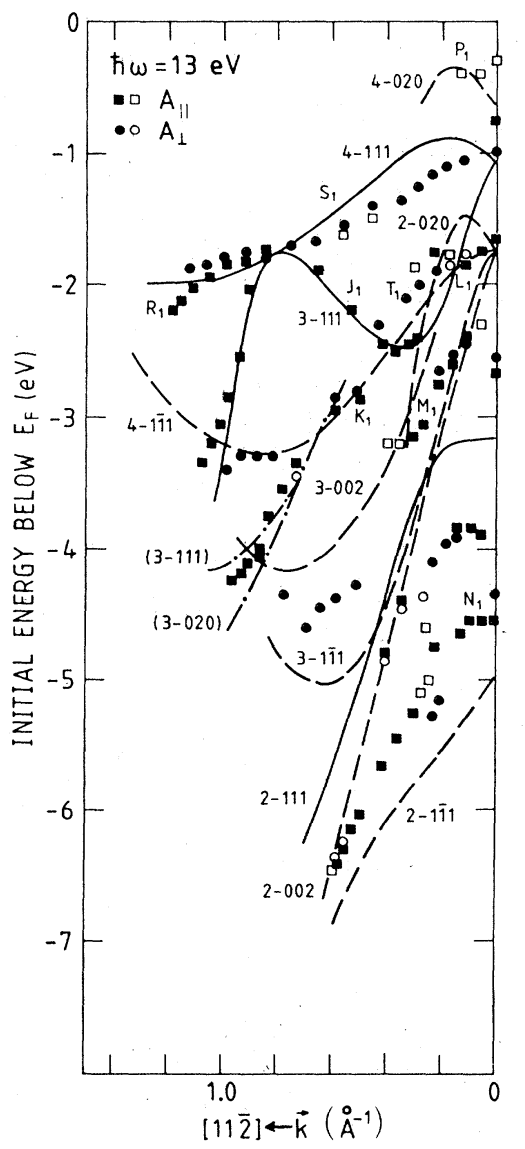

FIG. 8. Theoretical $E_{i}\left(\mathbf{k}_{\|}\right)$dispersions for calculated valence bands and several free-electron final bands. Dispersions corresponding to emission involving the primary (111) free-electron band are drawn as solid lines, while other dispersions along the [112] direction are marked with dashed lines. Transitions shown along this line calculated for the [2Ti1] direction are marked with dashed-dotted lines. Experimental points from Figs. 6 and 7 are shown with squares and circles, respectively. Solid and open symbols correspond to strong and weak (or uncertain) structures. The experimental dispersions $J_{1}$ and $S_{1}$ are in good agreement with the calculated (3-111) and (4-111) dispersions.

symbols correspond to strong and weak structures in the spectra. Solid dispersion curves correspond to transitions to the primary-cone free-electron band, the dashed lines to other free-electron bands. The dashed-dotted dispersion curves indicate dispersions along the [2 $\overline{1} \overline{1}]$ direction that, for symmetry reasons, can also be seen along the $[11 \overline{2}]$ direction. For the calculated initial-state dispersion curves, the actual transitions are indicated. For clarity, several calculated dispersion or parts of dispersion have been omitted in the figures.

The observed structures $\left(J_{1}\right)$ and $\left(S_{1}\right)$ in Figs. 6 and 7 are found to correspond to transitions from bands $3(+)$ and $4(-)$, respectively, to the (111) primary-cone band. These transitions are indicated by the notation (3-111) and (4-111). The structure $\left(K_{1}\right)$ dispersing downward in Fig. 6 follows the calculated disperson for transitions from

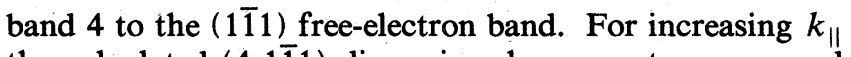

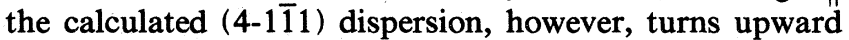
again, and no calculated dispersion for the [112] direction is present that corresponds to the rather strong downward-dispersing feature in the photoemission spectra. However, a transition calculated for one side of the [111] surface normal can also, in principle, be observed on the other side for the negative of the $k_{\|}$value, although in this case the group velocity of the final state is directed in the opposite direction. The transitions (3-111) and (3-020) for $k_{\|}$along the [2 $\overline{1} \overline{1}$ ] direction are shown with dasheddotted lines for the negative of their calculated $k_{\|}$values in Fig. 8. It is therefore possible that the dispersion of structure $K_{1}$ is explained by one of these transitions. The structure $L_{1}$ close to normal emission can also be seen to follow the (4-111) transition, although some other transitions are present in this region. The dispersion of structure $M_{1}$ cannot definitely be assigned to any transition, since there are several calculated dispersions of similar slope in this region. Three more features are worth noting, namely structures $N_{1}$ and $P_{1}$, which are likely to correspond to the transitions (2-111) and (4-020), and structure $R_{1}$, which is coincident with structure $S_{1}$ in Fig. 7 and corresponds to transitions from band 4 to the (111) free-electron band. A clear structure $\left(T_{1}\right)$ in Fig. 7 is seen to follow the calculated (4-111) transition rather well.

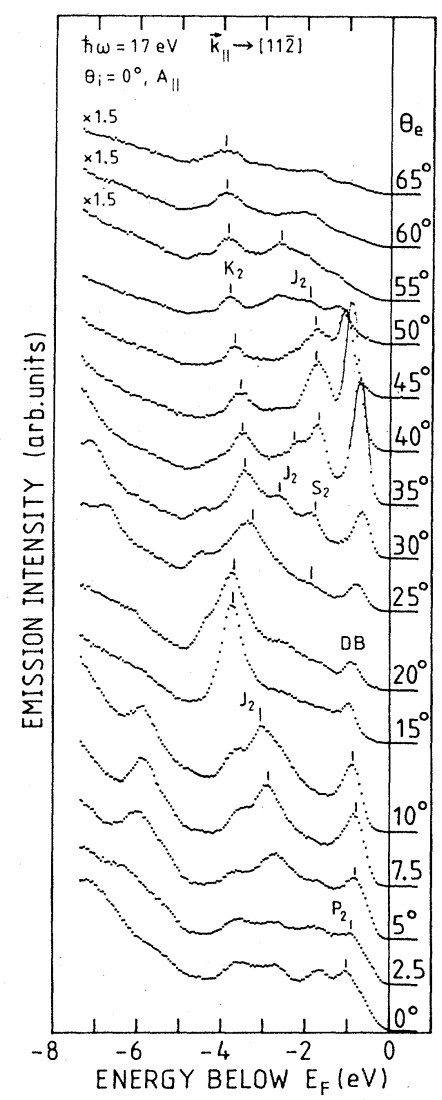

FIG. 9. Photoemission spectra for various emission angles for the $\theta_{i}=0^{\circ}, A_{\|}$geometry at $\hbar \omega=17 \mathrm{eV}$ along the [112] direction. DB corresponds to emission from the dangling bond band. 
Apart from $S_{1}$ and $T_{1}$, a number of structures are present in Fig. 7. Most are, however, visible over small $k_{\|}$regions only and cannot be associated definitely with the calculated dispersions (see Fig. 8).

At a photon energy of $17 \mathrm{eV}$, spectra were recorded along the [112] direction for the $\theta_{i}=0^{\circ}, A_{\|}$geometry and along the $[2 \overline{1} \overline{1}]$ direction for $\theta_{i}=0^{\circ}, A_{\|}$(see Figs. 9 and 10). Along the [112] direction, similar features are present in the spectra for $\hbar \omega=17 \mathrm{eV}$ as were seen for $\hbar \omega=13 \mathrm{eV}$. A rapidly dispersing structure $\left(J_{2}\right)$ corresponding to transitions from band 3 to the (111) freeelectron band is present, as well as a part of the flatter dispersion for the (4-111) transitions $\left(S_{2}\right)$ (see Fig. 11). Also at this photon energy a structure $\left(K_{2}\right)$ splits off from the (3-111) peak $\left(J_{2}\right)$ and disperses downward, although more slowly than for $\hbar \omega=13 \mathrm{eV}$ (Fig. 6). As for $13 \mathrm{eV}$, the observed peak is close to calculated transitions along the [ $2 \overline{1} \overline{1}$ ] line for the larger angles [transitions from band 3 to the (020) free-electron band, as shown by a dashed-dotted line along the [112] direction]. At a binding energy of $\approx-1 \mathrm{eV}$, a pronounced structure $\left(P_{2}\right)$ close to the (4-020) transition is observed. In spectra recorded along the [2 $\overline{1} \overline{1}]$ direction (Fig. 10), many structures are present. The dominating feature $\left(U_{2}\right)$ disperses down from $\approx-1.45 \mathrm{eV}$ at normal emission to $\approx-3.4 \mathrm{eV}$ at $\theta_{e}=45^{\circ}$. At high angles, a structure $V_{2}$ splits off from

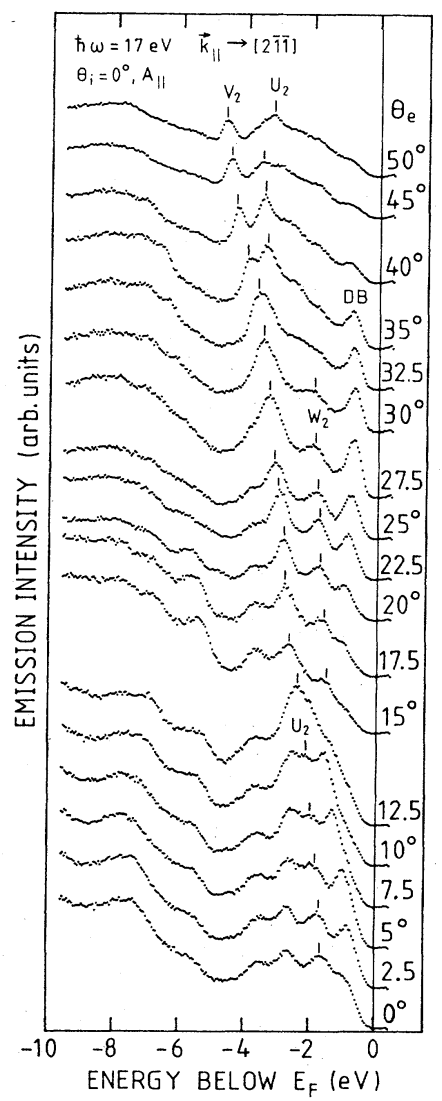

FIG. 10. Photoemission spectra for various emission angles for the $\theta_{i}=0^{\circ}, A_{\perp}$ geometry at $\hbar \omega=17 \mathrm{eV}$ along the [112] direction. DB corresponds to emission from the dangling bond band.

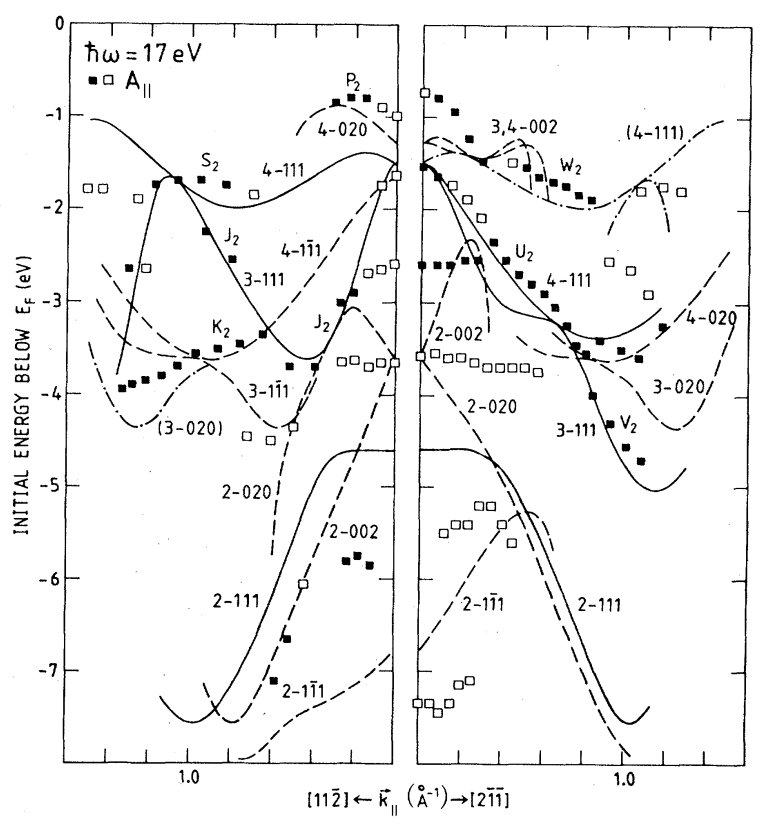

FIG. 11. Theoretical and experimental $E_{i}\left(\mathbf{k}_{\|}\right)$dispersions at

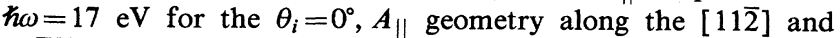
$\left[\begin{array}{lll}2 & \overline{1} & \overline{1}\end{array}\right]$ directions.

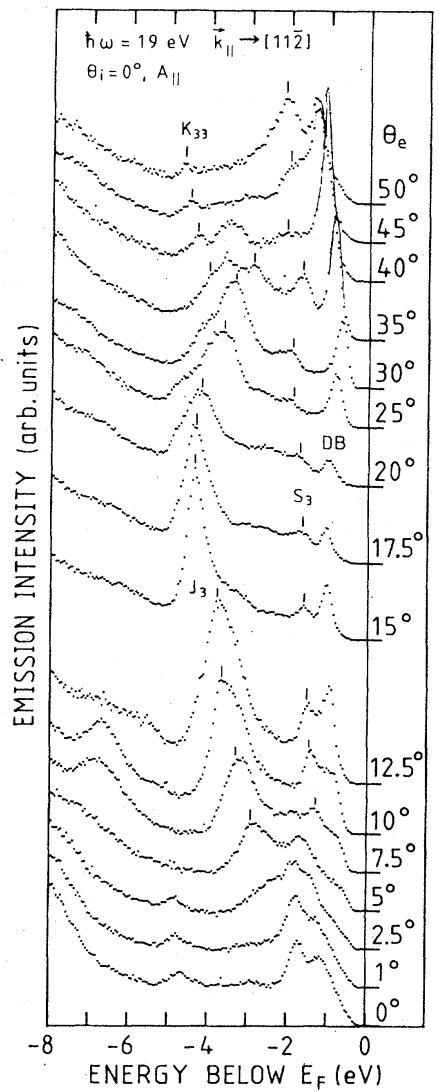

FIG. 12. Theoretical and experimental $E_{i}\left(\mathbf{k}_{\|}\right)$dispersions at $\hbar \omega=17 \mathrm{eV}$ for the $\theta_{i}=0^{\circ}, A_{\|}$geometry along the [112] and [2 $1 \overline{1}$ ] directions. DB corresponds to emission from the dangling bond band. 
$U_{2}$ toward higher binding energies. The corresponding dispersions for these structures (Fig. 11) can be seen to agree rather well with the calculated dispersions for transitions from bands 3 and 4 to the (111) free-electron band. The dispersions for other structures in Fig. 10 have also been plotted in Fig. 11. However, these cannot be identified directly with calculated dispersions. The slowly dispersing structure $\left(W_{2}\right)$ at $\approx-1.7 \mathrm{eV}$ is not in agreement with any calculated dispersion for the [2 $\overline{1} \overline{1}]$ direction, but it is close to the dispersion (4-111) for the [11 2 ] direction shown by a dashed-dotted line. In the highbinding-energy region, some structures have shapes similar to calculated dispersions, although the agreement in energy is poor.

At a photon energy of $19 \mathrm{eV}$, spectra were recorded along the [115] direction for the $A_{\|}$(Fig. 12) and $A_{\perp}$ geometries (not shown) and along the [2 $\overline{1} \overline{1}]$ direction for the $A_{\|}$case (not shown). The corresponding dispersion plots are shown in Fig. 13. For the [11 $\overline{2}]$ direction, a strong peak $\left(J_{3}\right)$ is again present for the transition from band 3 to the (111) free-electron band, and in this case the downward-sloping part is clearly visible, mainly for the $A_{\|}$geometry. The dispersion of structure $S_{3}$ has a slope similar to the calculated (4-111) dispersion, although positioned $0.4 \mathrm{eV}$ too high. This structure could, however, also correspond to the (4-020) transition. At large $k_{\|}$ values some structures split off from the $J_{3}$ structure, dispersing upward $\left(K_{31}\right)$ and downwards $\left(K_{32}\right.$ and $\left.K_{33}\right)$. Structures $K_{32}$ and $K_{31}$, observed for the $A_{\perp}$ case, are close to the transitions $(3-1 \overline{1} 1)$ and (4-1 $\overline{1} 1)$, respectively, whereas structure $K_{33}$ is close to the (3-111) transition for the $[2 \overline{1} \overline{1}]$ direction (dashed-dotted line). However, the (3-111) transition for the [2 $\overline{1} \overline{1}]$ direction is reproduced by emission along that line (see Fig. 13, structure $V_{3}$ ), with a slightly different dispersion. The dominating peak in the emission along the [2 $\overline{1} \overline{1}]$ direction is the downward-

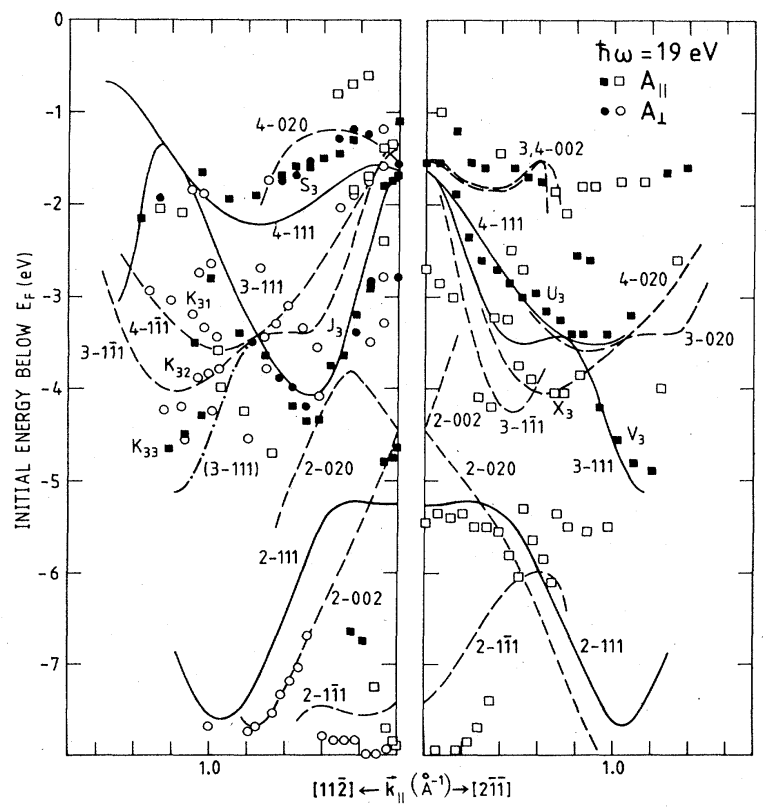

FIG. 13. Theoretical and experimental $E_{i}\left(\mathbf{k}_{\|}\right)$dispersions at $\hbar \omega=19 \mathrm{eV}$ for the $A_{\|}$and $A_{\perp}$ geometries. sloping structure $\left(U_{3}\right)$ close to the calculated dispersion for the (4-111) transition. At large angles, a structure $\left(V_{3}\right)$ is present close to the dispersion of the (3-111) transition. In the spectra for the [2 $\overline{1} \overline{1}]$ direction, a number of weak structures whose origin cannot be unambigously determined are present, while in the high-binding-energy region the dispersions of some structures have the same shape as the calculated dispersions.

For the off-normal measurements described so far, the angle of incidence has been either $\theta_{i}=0^{\circ}$ or $\theta_{i}=15^{\circ}$. In a previous experiment, ${ }^{37}$ the cleaved surface of germanium was studied with unpolarized light from a resonance lamp, $\hbar \omega=16.8 \mathrm{eV}$, and $\hbar \omega=21.2 \mathrm{eV}$ at an angle of incidence of $\theta_{i}=45^{\circ}$. The general appearance of the spectra obtained in these measurements is very different from those obtained with polarized light. In Fig. 14 spectra obtained with unpolarized light at $\hbar \omega=21.2 \mathrm{eV}$ are shown. They were recorded along the [ $1 \overline{2} 1]$ direction for an angle of incidence $\theta_{i}=45^{\circ}$. In this case, therefore, transitions sensitive to the component of the electric field vector perpendicular to the surface should be enhanced. The emission direction was chosen relative to the $2 \times 1 \mathrm{SBZ}$ so that the $\bar{\Gamma}-\bar{J}^{\prime}$ direction of the surface was probed simultaneously. The dangling-bond surface state is visible in most spectra and reveals an almost flat dispersion in this direction. However, the surface state is close to the projected bulk bands for most angles and care must be taken in

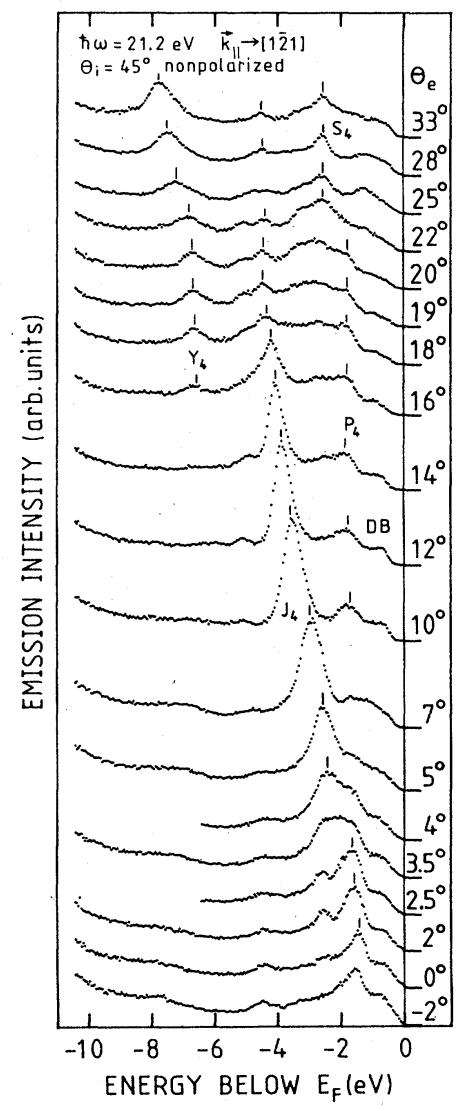

FIG. 14. Photoemission spectra for various emission angles recorded with nonpolarized light at $\hbar \omega=21.2 \mathrm{eV}$ for $\theta_{i}=45^{\circ}$. DB corresponds to emission from the dangling bond band. 
separating this state from the background. At angles of about $12^{\circ}$ where the surface state is clearly in the projected band gap at the $\bar{J}^{\prime}$ point, the initial energy is $\approx-0.65$ eV. A rapidly dispersing peak $\left(J_{4}\right)$ dominating the spectra over a wide range of angles has a dispersion in close agreement with the calculated dispersion for the (3-111) transition (see Fig. 15). Some emission is found close to the (4-111) transition $\left(S_{4}\right)$, and the (4-020) transition is also present $\left(P_{4}\right)$. The spectra for $\theta_{e}=0^{\circ}$ resemble the normal-emission spectra at $\hbar \omega=21 \mathrm{eV}$ in Fig. 2, and structure $P_{4}$, which probably contributes to the normal emission, may originate from the $L_{3}^{\prime}$ point, as discussed for the normal-emission spectra. At large emission angles, a dispersive peak $\left(Y_{4}\right)$ at high binding energies increases in intensity. This peak is likely to be due to transitions to the primary free-electron band, but this time from the second valence band.

For measurements at $\hbar \omega=25 \mathrm{eV}$, the stronger features in the spectra are in agreement with calculated dispersions for transitions to the primary free-electron band, and

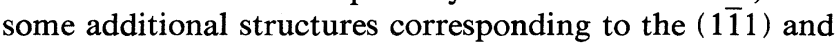
(020) free-electron bands are also present. In a previous publication, ${ }^{14}$ spectra obtained with polarized light in the [101] direction for photon energies of 21.2 and $35 \mathrm{eV}$ were presented. At $21.2 \mathrm{eV}$ the dominating bulk contribution in these spectra, a rapidly dispersing peak, has a dispersion in good agreement with the calculated dispersion of the (3-111) transition in the $(1 \overline{2} 1)$ plane. At $\hbar \omega=35 \mathrm{eV}$ the bulk features are broader and the experi-

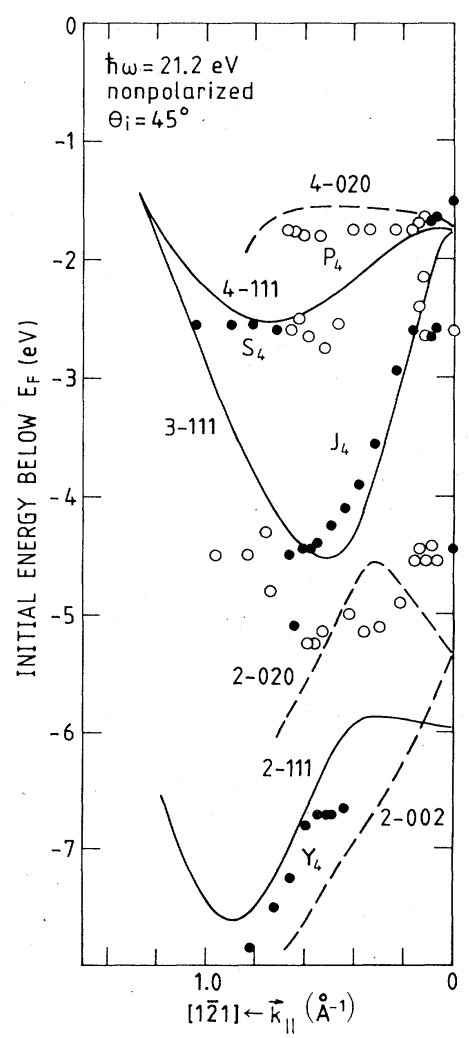

FIG. 15. Theoretical and experimental $E_{i}\left(\mathbf{k}_{\|}\right)$dispersions at $\hbar \omega=21.2 \mathrm{eV}$ along the [ $1 \overline{2} 1]$ direction. mental dispersions more uncertain; nonetheless, dispersions in rather good agreement with the (3-111) and (4-111) transitions are observed.

\section{DISCUSSION}

In early studies of the cleaved surface of germanium by Grobman et al. ${ }^{8}$ angle-integrated measurements of the $\mathrm{Ge}(111) 2 \times 1$ surface were performed with synchrotron radiation for photon energies between 6.5 and $25 \mathrm{eV}$. A surprisingly detailed identification of the spectral features with theoretical bulk bands and calculated spectra was possible using a direct-transition model of photoemission. The positions of several critical points in the band structure were determined and dispersive peaks were associated with direct transitions between valence and conduction bands from pseudopotential calculations. The first angleresolved photoemission experiment on the Ge(111) surface was performed by Bringans and Höchst ${ }^{15}$ for the annealed $c-(2 \times 8)$ surface at a photon energy of $21.2 \mathrm{eV}$. Some bulk features were found to have dispersions in qualitative agreement with calculated dispersions for a (111) freeelectron final band. This experiment was followed by angle-resolved measurements on the cleaved $(111) 2 \times 1$ surface $\mathrm{e}^{12-14}$ and a further study of the $c-(2 \times 8)$ surface. $^{16}$ These later studies dealt with the surface-state distributions and the bulk bands were not discussed in detail. Nelson et al. presented the first angle-resolved measurements of the $\mathrm{Ge}(001) 2 \times 1$ surface. ${ }^{17,18}$ Synchrotron radiation in the energy range $10-29 \mathrm{eV}$ was used, and in normal-emission spectra two features were identified with transitions between valence and conduction bands along the $\Gamma-X$ line in the BZ from an empirical pseudopotential calculation. A similar study was performed by Hsieh et al. ${ }^{20}$ for the $\mathrm{Ge}(001) 2 \times 1$ surface, but for a wider photon-energy range $(14-45 \mathrm{eV})$. In this case a method previously applied to the GaAs(110) surface with a freeelectron final band was used to determine the initial-state bands. ${ }^{30}$ Once again, two features in the spectra were associated with valence bands along the $\Gamma-X$ line.

In the angle-resolved work carried out so far, only one final-state band for the emission has been used, or only normal-emission data have been interpreted in studying the bulk band structure. In a more detailed analysis, the application of several final bands is needed for a better description of the real band structure, and off-normalemission data permit a detailed study of initial-state dispersions.

In the present analysis it is suggested that transitions contributing to the emission have pronounced matrix elements when the final states are close to free-electron bands. However, from comparison with calculated normal-emission spectra, it is concluded that contributing real final-state bands exist that are not in the vicinity of free-electron bands. It is possible that these states can be described with free-electron bands of different effective mass or with different inner potentials, but such bands cannot simultaneously account for the off-normalemission spectra.

From the present normal-emission data, transitions close to the $(1 \overline{1} 1)$ free-electron band are observed, as are 
transitions to a higher positioned band not described by free-electron bands for the present choice of the inner potential (as determined from off-normal-emission data). Transitions to the primary (111) free-electron band are not observed in normal-emission spectra. Further, the calculated $\Lambda_{1}$ bands are not in the immediate vicinity of this free-electron band, which could account for the large difference observed from a similar experiment on the $\mathrm{Si}(111) 2 \times 1$ surface, ${ }^{32}$ where an experimental final band close to the primary-cone free-electron band was observed. In the case of $\mathrm{Si}$, the (111) free-electron band is closer to the calculated $\Lambda_{1}$ bands, and the gaps between these are smaller than for Ge.

In light of the above discussion, the choice of inner potential for the assumed free-electron bands is crucial. The position of the free-electron bands is determined from the agreement of the calculated off-normal dispersions for these final bands with experimentally obtained dispersions. In this determination transitions from bands 3 and 4 were used, and the calculated transitions were found to be very sensitive to the value of the inner potential. In addition, a lowering of bands 3 and 4 was necessary to describe accurately the observed transitions as transitions to free-electron final bands. In general, good agreement is observed in this application of free-electron-like final bands. In spectra the strongest features correspond to primary-cone emission, but substantial secondary-cone emission is also present. However, it is evident that the assumption of free-electron-like final bands does not account for all the details observed in the spectra, and deviations from the calculated initial-state dispersion are quite frequent. For a more complete interpretation of the experimental data, a comparison with calculated photoemission spectra for off-normal directions is necessary. Some encouraging results have been obtained for $\mathrm{Ge}$ and $\mathrm{Si}$ in a recent study by Persson et al. ${ }^{36}$

Hsieh et al. ${ }^{20}$ used an inner potential of $7.7 \mathrm{eV}$ for a free-electron band in interpreting normal-emission data from the $\mathrm{Ge}(001) 2 \times 1$ surface. The valence bands determined in this case were in rather good agreement with calculated bands. In applying this inner potential to the free-electron bands in the present study, the resulting calculated initial-state dispersions were positioned far too high in energy and agreement with experimental dispersions was poor. The primary-cone free-electron band by Hsieh et al. is found to be close to a calculated final band by Nelson et al. ${ }^{18}$ and to a band in the band calculation used in the present study (see Fig. 1). It is notable that this band is a continuation in the $\Gamma-X$ direction of the bands $15+18$ in the $\Gamma-L$ direction which were found to contribute to the emission in the normal-emission spectra. Approximating the calculated "primary-cone" band in the $\Gamma-X$ direction with a free-electron band is probably less sensitive to the choice of inner potential since in this direction the bands are very steep. A possible error in the energy of the final band in the $\Gamma-X$ direction then produces a smaller error in the determined $k_{\perp}$ value than would be the case for a less steep band in the $\Gamma$ - $L$ direction.

The use of polarized synchrotron radiation allows us, in principle, to separate initial states of different parity with respect to the (110) mirror plane. ${ }^{1}$ This effect may be clearly seen, e.g., for off-normal emission at $\hbar \omega=13 \mathrm{eV}$, Fig. 8, where transitions from band $3(+)$ and band $4(-)$ are pronounced for the $A_{\|}$and $A_{\perp}$ polarization geometries, respectively. However, in several cases some emission from a band of different parity than that of the light is present in the spectra. As discussed in the literature, $1,38,39$ the spin-orbit interaction mixes states of different parity. In a similar experiment on $\mathrm{Si}^{32}$ using the same experimental setup, no emission from bands of opposite parity to that of the light was observed. It is therefore likely that the larger spin-orbit interaction present in germanium is responsible for the observed effects. In addition, the finite angle resolution $\left( \pm 2^{\circ}\right)$ of the analyzer may contribute to the emission from the opposite-parity band, since points in the BZ not in the mirror plane are probed simultaneously. Fully relativistic calculations of the matrix elements of these transitions are needed to clarify this point. As discussed previously, the observed differences in initial energy for the peaks $C$ in the normal-emission series (Figs. 3 and 4) may correspond to the spin-orbit splitting of bands 3 and 4 along the $\Gamma-L$ line, in agreement with the calculated value for this split, $\approx 0.2 \mathrm{eV}^{26}$

For a wide range of photon energies a number of nondispersive structures are present in normal- and offnormal-emission spectra. These probably correspond to emission from critical points along different symmetry lines in the BZ, which indicates that not only direct transitions, but density-of-states effects, are important in photoemission from Ge.

For the $\pi$-bonded-chain model of the Ge(111) $2 \times 1$ surface, ${ }^{40,41}$ a surface state associated with $\sigma$ bonds between surface atoms has been proposed. ${ }^{41}$ This state disperses in a projected bulk band gap $\approx 8 \mathrm{eV}$ below the valence-band maximum in the $\bar{\Gamma}-\bar{J}$ direction of the SBZ. At these high binding energies this direction was investigated with polarized light at angles of incidence $\theta_{i}=0^{\circ}$ and $\theta_{i}=45^{\circ}$ for photon energies of 21.2 and $25 \mathrm{eV}$, and with unpolarized light for photon energies of 16.8 and $21.2 \mathrm{eV}\left(\theta_{i}=45^{\circ}\right)$. However, apart from the previously mentioned danglingbond surface state, no further signs of surface states have been noticed.

\section{CONCLUSIONS}

In photoemission spectra recorded for a wide range of photon energies, several structures associated with direct transitions to free-electron-like final bands are observed. The strongest contributions correspond to primary-cone emission, but several secondary-cone transitions have also been identified. For a best fit to the experimental data, the uppermost pair of valence bands have been lowered and the experimental $L_{3}^{\prime}$ point is positioned at $-1.74 \mathrm{eV}$ below $E_{V}, 0.35 \mathrm{eV}$ lower than in a LAPW band calculation. In normal-emission spectra no transitions involving the (111) primary free-electron band are observed. The calculated $\Lambda_{1}$ bands are not found in the immediate vicinity of this free-electron band, which perhaps can explain why free-electron-like behavior is not observed in the nor- 
mal direction, as is the case for $\mathrm{Si}$, where the $\Lambda_{1}$ bands are closer to the free-electron band ${ }^{32}$ in this direction. By comparison with calculated spectra ${ }^{36}$ for normal emission, it is further concluded that contributing final-state bands not described by free-electron bands must also be present.

\section{ACKNOWLEDGMENTS}

We wish to thank Dr. N. Ueno for his help during the measurements. This work was supported by the Swedish Natural Science Research Council.
*Present address: IBM Zurich Research Laboratory, 8803 Rüschlikon, Switzerland.

${ }^{\dagger}$ Present address: Fritz-Haber-Institut der Max-PlanckGesellschaft, 1000 Berlin 33, Federal Republic of Germany.

${ }^{1}$ G. W. Gobeli, F. G. Allen, and E. O. Kane, Phys. Rev. Lett. 12, 94 (1964).

${ }^{2}$ D. E. Eastman and W. D. Grobman, Phys. Rev. Lett. 28, 1378 (1972).

${ }^{3}$ R. A. Pollak, L. Ley, S. Kowalczyk, D. A. Shirley, J. D. Joannopoulos, D. J. Chadi, and M. L. Cohen, Phys. Rev. Lett. 29, 1103 (1972).

${ }^{4}$ W. D. Grobman and D. E. Eastman, Phys. Rev. Lett. 29, 1508 (1972).

${ }^{5}$ D. E. Eastman, W. D. Grobman, J. L. Freeouf, and M. Erbudak, Phys. Rev. B 9, 3473 (1974).

${ }^{6}$ W. D. Grobman and D. E. Eastman, Phys. Rev. Lett. 33, 1034 (1974).

${ }^{7}$ T. Murotani, K. Fujiwara, and M. Nishijima, Phys. Rev. B 12, 2424 (1975).

${ }^{8}$ W. D. Grobman, D. E. Eastman, and J. L. Freeouf, Phys. Rev. B 12, 4405 (1975).

9J. E. Rowe, Solid State Commun. 17, 673 (1975)

10J. E. Rowe, Surf. Sci. 53, 461 (1975).

${ }^{11}$ F. J. Himpsel, D. E. Eastman, P. Heimann, B. Reihl, C. W. White, and D. M. Zehner, Phys. Rev. B 24, 1120 (1981).

12J. M. Nicholls, G. V. Hansson, R. I. G. Uhrberg, and S. A. Flodström, Phys. Rev. B 27, 2594 (1983).

${ }^{13}$ F. Solal, G. Jezequel, A. Barski, P. Steiner, R. Pinchaux, and Y. Petroff, Phys. Rev. Lett. 52, 360 (1984).

14J. M. Nicholls, G. V. Hansson, U. O. Karlsson, R. I. G. Uhrberg, R. Engelhardt, K. Seki, S. A. Flodström, and E.-E. Koch, Phys. Rev. Lett. 52, 1555 (1984).

${ }^{15}$ R. D. Bringans and H. Höchst, Phys. Rev. B 25, 1081 (1982).

${ }^{16}$ T. Yokotsuka, S. Kono, S. Suzuki, and T. Sagawa, J. Phys. Soc. Jpn. 53, 696 (1984); Jpn. J. Appl. Phys. 23, L69 (1984).

17J. G. Nelson, W. J. Gignac, R. S. Williams, S. W. Robey, J. G. Tobin, and D. A. Shirley, Phys. Rev. B 27, 3924 (1983).

18J. G. Nelson, W. J. Gignac, R. S. Williams, S. W. Robey, J. G. Tobin, and D. A. Shirley, Surf. Sci. 131, 290 (1983).

${ }^{19}$ S. D. Kevan and N. G. Stoffel, Phys. Rev. Lett. 53, 702
(1984).

${ }^{20}$ T. C. Hsieh, T. Miller, and T.-C. Chiang, Phys. Rev. B 30, 7005 (1984)

${ }^{21}$ D. E. Eastman and J. L. Freeouf, Phys. Rev. Lett. 33, 1601 (1974); J. L. Freeouf and D. E. Eastman, CRC Crit. Rev. Solid State Sci. 5, 245 (1975).

${ }^{22}$ G. M. Guichar, G. A. Garry, and C. A. Sébenne, Surf. Sci. 85, 326 (1979).

${ }^{23}$ D. Straub, L. Ley, and F. J. Himpsel (unpublished).

24J. R. Chelikowsky and M. L. Cohen, Phys. Rev. B 14, 556 (1976).

${ }^{25}$ C. S. Wang and B. M. Klein, Phys. Rev. B 24, 3393 (1981).

26P. E. S. Persson (unpublished).

${ }^{27}$ G. D. Mahan, Phys. Rev. B 2, 4334 (1970).

${ }^{28}$ L. Hedin and B. I. Lundqvist, J. Phys. C 4, 2064 (1971).

${ }^{29}$ P. Thiry, D. Chandesris, J. LeCante, C. Guillot, P. Pinchaux, and Y. Petroff, Phys. Rev. Lett. 43, 82 (1979).

${ }^{30}$ T.-C. Chiang, J. A. Knapp, M. Aono, and D. E. Eastman, Phys. Rev. B 21, 3513 (1980).

${ }^{31}$ F. Solal, G. Jezequel, F. Houzay, A. Barski, and R. Pinchaux, Solid State Commun. 52, 37 (1984).

${ }^{32}$ R. I. G. Uhrberg, G. V. Hansson, U. O. Karlsson, J. M. Nicholls, P. E. S. Persson, S. A. Flodström, R. Engelhardt, and E.-E. Koch, Phys. Rev. Lett. 52, 2265 (1984); Phys. Rev. B 31, 3795 (1985).

${ }^{33}$ C. A. Feldmann, R. Engelhardt, T. Permien, E.-E. Koch, and V. Saile, Nucl. Instrum. Methods 208, 785 (1983).

34J. Hermanson, Solid State Commun. 22, 9 (1977).

${ }^{35}$ W. Eberhardt and F. J. Himpsel, Phys. Rev. B 21, 5572 (1980).

36P. E. S. Persson, R. I. G. Uhrberg, and J. M. Nicholls (unpublished).

37J. M. Nicholls (unpublished).

38J. Anderson, G. J. Lapeyre, and R. J. Smith, Phys. Rev. B 17, 2436 (1978).

${ }^{39}$ H. Przybylski, A. Baalmann, G. Borstel, and M. Neumann, Phys. Rev. B 27, 6669 (1983).

40K. C. Pandey, Phys. Rev. Lett. 47, 1913 (1981).

41J. E. Northrup and M. L. Cohen, Phys. Rev. B 27, 6553 (1983). 